\title{
High and low anxiety and GSR adaptation
}

ROBERT FRIED, MIRIAM FRIEDMAN AND LIVINGSTON WELCH HUNTER COLLEGE OF THE CITY UNIVERSITY OF NEW YORK ${ }^{1}$

GSR records were obtained for two groups of 20 Ss differing in score on the Eysenck Personality Inventory (high and low scores) while they were presented with a series of 80 stimuli four different lights alternating in apparent random sequence). Typical adaptation phenomena were observed for the high- $N$ group while no adaptation was evident in the low$N$ group. It is concluded that the observed relationship between anxiety and adaptation supports the contention that adaptation of the GSR is a form of conditioned inhibition.

It has been shown, in previous studies, that the introduction of a novel stimulus (NS) during adaptation of the galvanic skin response (GSR) to sequential visual stimuli does not always result in a significant change in the course of adaptation (Fried, Korn, \& Welch, 1966; Fried, Welch, \& Friedman, 1966; Fried, Welch, Friedman, \& Gluck, 1967). The relationship between NS which alter adaptation and those which do not suggests that adaptation of the GSR manifests a form of conditionedinhibition analogous to that usually observed in prolonged psychomotor tasks.

This interpretation of GSR adaptation is suggested as an alternative to the usual fatigue-effects explanation of the phenomenon. And, if the conditioned inhibition interpretation of GSR adaptation is correct, it offers a methodological approach to the study of stimulus novelty which traditional approaches lack: Observation of an orienting response (OR) or its disappearance hardly provides a useful paradigm for parametric investigation of the dimension novelty.

The failure to observe the effects of a NS, 1.e., a stimulus not a part of the adaptation series, reported by the present investigators, has been ascribed to the use of basal resistance or electrodermal level (EL) rather than the more conventional transient changes. This criticism by Furedy (in press) is difficult to evaluate since his procedure is entirely different from the one the present investigators have reported and provides, therefore, no real basis for comparison of the data.

It should be pointed out, however, that there is no way, at present, to determine whether a transient, in a given $S$, for a given situation, is signal or noise. The unreliability of the transient change in skin resistance necessitates composite "curves" which average the response measure for considerable numbers of Ss. These composites are often the result of response sampling somewhere within a given time span following stimulus presentation (often, it is the sharpest rise within a time span ranging from 3 to $5 \mathrm{sec})$. This procedure, when investigators justify it, is based on a large number of assumptions, none of which has an empirical basis.

Since there is no basis for extricating transient change from the EL, the strategy of the present investigators has been, in the past, to use only the EL at fixed time periods as an indicator of a change in activation level (Woodworth \& Schlosberg, 1954). This strategy, for which the present investigators have been taken to task, results in more nearly total response measures, over time, than does the use of the "more conventional" transient change. The method is considerably more reliable, although it is less sensitive to individual response differences. In return, the procedure also attenuates the need for weighting transients with respect to the magnitude of change and EL for Ss with often vastly different ELs. It is by no means suggested that this procedure be employed in all GSR monitoring situations, but only that it is the most appropriate one under the prevailing conditions.

In previous studies, cited above, it has been suggested that NS which result in an OR during an adaptation series function as disinhibiting stimuli (in the classical conditioning sense). The contention that physiological adaptation of the GSR is a form of conditioning rather than fatigue would be strengthened if it can be shown that adaptation is a pattern predictable by conditioning theory.

Preliminary findings indicate that the OR is observed only when the NS is sufficiently distant (on a generalization gradient) from the adaptation stimuli so that, although the adaptation stimuli are perceptually discriminable, generalization does not take place from one to the other (Fried, Welch, Friedman, \& Gluck, 1967a).

The determination that the observed relationship between anxiety and conditionability also applies to adaptation phenomena would help to strengthen the position that adaptation is a form of conditioning. Pavlov's excitation and inhibition processes (1955) provide the basis for the contention by Franks (1961) that anxious Ss should condition more readily than non-anxious Ss.

A significant positive relationship has been shown to exist between anxiety and GSR conditionability (Becker \& Matheson, 1961), though these findings have not always been corroborated (Davidson, Payne, \& Sloan, 1961). However, the manipulation of anxiety levels, and associated changes in GSR, have been demonstrated to be a practical procedure (Wolpe, Lazarus, \& Fried, 1967). 


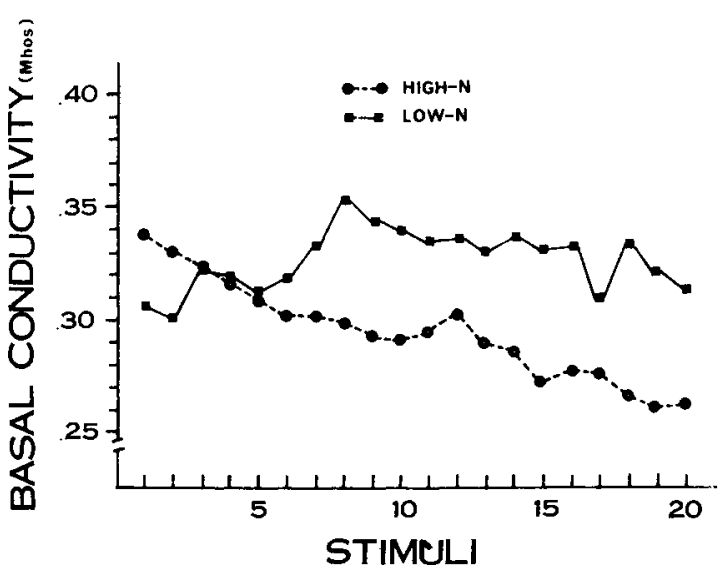

Fig. 1. GSR adaptation: mean amplitude (mhos) on presentation of the amber light stimulus.

The aim of the present study is to determine whether differences in GSR adaptation patterns correspond to differences in anxiety levels in human Ss. Method

Subjects. Twenty college undergraduates were assigned to each of two groups on the basis of high $(+1.00 \sigma$ at least) and low (-1.00 $\sigma$ at least) scores on the Eysenck Personality Inventory (Form B).

Apparatus. The GSR records were obtained with a custom designed constant voltage bridge coupled to a Bausch and Lomb recorder. The visual stimuli, four $60 \mathrm{~W}$ lamps shielded with cellulose acetate filters (blue, green, white, and amber) were presented through a common aperture, $1-1 / 2$ in. in diameter in the apparatus housing. The stimuli appeared to the $S$ as a changing circular patch of light. The sequence of hues appeared random and had an average probability of .25 for each hue. Stimulus duration was $7.5 \mathrm{sec}$, and interstimulus time was $.01 \mathrm{sec}$.

Each $S$ was seated in a darkened room with GSR electrodes attached to the index and middle finger of the left hand. Ten minutes were allowed to elapse before the stimuli were presented and adaptation begun.

Results

The data consist of conductance measures at the termination of the presentation of each amber light stimulus. For each $\mathrm{S}$, there were 20 such presentations. These data are summarized in Fig. 1.

The trends are significantly different $(\alpha=.01)$. The systematic decrease in EL indicates general response decrease to the adapting stimuli, as well as the transients, in the high-N group, while no such change was observed in the low-N group.

\section{Discussion}

The difference between the high- $\mathrm{N}$ and low-N groups in activation level in conjunction with the presentation of the adaptation stimuli conforms to the stated hypothesis. The high-N Ss adapted readily, while the low-N Ss showed no such decrease in activation level. Decrease of basal level without accompanying decrease in transient is extremely remote and likewise, failure to obtain change in EL also seldom occurs in conjunction with significant systematic change in transients.

It is concluded that the course of physiological adaptation is, in part, predictable from information concerning conditionability. And this evidence, in conjunction with previous findings, strengthens the contention that adaptation is a form of conditioned inhibition of responses to the adaptation stimuli.

\section{References}

BECKER, W. C., \& MATHESON, H. H. GSR conditioning, anxiety, and extroversion. J. abnorm. soc. Psychol., 1961, 62, 427-430.

FRANKS, C. M. Conditioning and abnormal behavior. In Eysenck, H. J. Handbook of Abnormal Psychology. New York: Basic Books, Inc., 1961, 460-467.

FRIED, R., KORN, S., \& WELCH, L. Effect of change in sequential visual stimuli on GSR adaptation. $J$. exp. Psychol., 1966, 72, 325-327.

FRIED, R., WELCH, L., \& FRIEDMAN, M. Stimulus novelty and intraseries primacy in GSR adaptation. Percept. Psychophys., 1966, 1, 345-346.

FRIED, R., WELCH, L., FRIEDMAN, M., \& GLUCK, S. Is no stimulus a stimulus? J. exp. Psychol., 1967, 73, 145-146.

FRIED, R., WELCH, L., FRIEDMAN, M., \& GLUCK, S. The effect of change in sequential visual stimuli: II, the novel stimulus as a disinhibiting stimulus. Percept. \& Psychophys., 1967, in press.

FUREDY, J. J. Novelty and the measurement of the GSR. J. exp. Psychol, in press.

PAVLOV, I. P. Selected Works (Trans. by S. Belsky). Moscow: Foreign Languages Publishing House, 1955, 315-344.

WOLPE, J., LAZARUS, A., \& FRIED, R. Psychophysiological correlates of systematic desensitization of phobias. Read before the Amer. Psychiat. Assoc. annual meeting, May 1967.

Note

1. Also Department of Psychiatry, Cornell University Medical College. 